\title{
Telehealth Use by Age and Race at a Single Academic Medical Center During the COVID-19 Pandemic: Retrospective Cohort Study
}

Jennifer P Stevens ${ }^{1}$, MD, MS; Oren Mechanic ${ }^{2}$, MD; Lawrence Markson ${ }^{3}$, MD; Ashley O'Donoghue ${ }^{1}$, PhD; Alexa B Kimball ${ }^{2}$, MD

\footnotetext{
${ }^{1}$ Center for Healthcare Delivery Science, Beth Israel Deaconess Medical Center, Boston, MA, United States

${ }^{2}$ Harvard Medical Faculty Physicians, Beth Israel Deaconess Medical Center, Boston, MA, United States

${ }^{3}$ Information Systems, Beth Israel Deaconess Medical Center, Boston, MA, United States
}

\section{Corresponding Author:}

Jennifer P Stevens, MD, MS

Center for Healthcare Delivery Science

Beth Israel Deaconess Medical Center

330 Brookline Avenue

Boston, MA, 02215

United States

Phone: 16176677165

Email: jpsteven@Bidmc.harvard.edu

\section{Abstract}

Background: During the COVID-19 pandemic, many ambulatory clinics transitioned to telehealth, but it remains unknown how this may have exacerbated inequitable access to care.

Objective: Given the potential barriers faced by different populations, we investigated whether telehealth use is consistent and equitable across age, race, and gender.

Methods: Our retrospective cohort study of outpatient visits was conducted between March 2 and June 10, 2020, compared with the same time period in 2019, at a single academic health center in Boston, Massachusetts. Visits were divided into in-person visits and telehealth visits and then compared by racial designation, gender, and age.

Results: At our academic medical center, using a retrospective cohort analysis of ambulatory care delivered between March 2 and June 10, 2020, we found that over half (57.6\%) of all visits were telehealth visits, and both Black and White patients accessed telehealth more than Asian patients.

Conclusions: Our findings indicate that the rapid implementation of telehealth does not follow prior patterns of health care disparities.

(J Med Internet Res 2021;23(5):e23905) doi: 10.2196/23905

\section{KEYWORDS}

access; barrier; cohort; COVID-19; demographic; equity of care; equity; outpatient; telehealth

\section{Introduction}

During the COVID-19 pandemic, many hospitals reduced in-person ambulatory care. Through payment parity and reductions in administrative barriers, including the Health Insurance Portability and Accountability Act of 1996 waivers by Health and Human Services, physicians rapidly began providing care through telemedicine $[1,2]$.
Our large academic physicians' group in Boston, Massachusetts, similarly expanded telehealth in mid-March 2020. This sudden move, however, risked exacerbating care inequity across racial and socioeconomic groups $[3,4]$. Given potential barriers faced by different populations [5], we investigated whether telehealth use was consistent and equitable across age, race, and gender. 


\section{Methods}

\section{Study Population and Data Source}

This study was deemed exempt by the institutional review board at the Beth Israel Deaconess Medical Center. In-person and telehealth visits to outpatient clinics staffed by our practicing faculty, between March 2 and June 10, 2020, for patients aged $\geq 18$ years were identified and compared to visits to identical clinics during the same period in 2019.

\section{Study Variables}

Patient age (in deciles), self-identified race, and gender were extracted from the electronic health records.

\section{Statistical Analysis}

Statistical tests were performed using Stata SE (version 14.2, StataCorp). In-person and telehealth use were stratified by week and race and compared to the same period in 2019. We conducted similar comparisons by gender and age deciles and explored interaction terms to determine whether different groups of age deciles and race used telehealth more or less than other groups. We further conducted a subgroup analysis of care provided after April 27; after this date, we had more complete documentation of whether the telehealth visit was conducted using video technology or over the telephone. In this subgroup, we explored whether older adults ( $\geq 65$ years old) made use of video technology at similar rates as younger patients. We similarly conducted a subgroup analysis during this period to investigate whether non-White patients used video technology at similar rates as White patients.

\section{Results}

Between March 2 and June 10, 2020, a total of 129,844 ambulatory visits were conducted, compared to 180,831 visits during the same period in 2019, which indicates a reduction of $28 \%$. Compared to 2019, visits in 2020 decreased for all racial groups (White patients: $31 \%$, Black patients: $23 \%$, Hispanic patients: $26 \%$, and Asian patients: $39 \% ; P<.001)$.

Among visits in 2020 , a total of 74,846 (57.6\%) visits were conducted through telehealth. Overall, Black $(n=9414$ of 15,423 , $61 \%)$ and White $(n=37,620$ of $63,397,59.3 \%)$ patients used telehealth rather than in-person visits at higher rates; Asian patients used telehealth the least $(n=3162$ of 5661, 55.9\%) (Figure 1). Patients with "unknown" racial designations displayed the lowest rates of telehealth use $(P<.001)$.

Figure 1. Comparison of outpatient visits in 2020 with those conducted during the same time period in 2019 (dotted line), by race at a single academic health system in Boston, Massachusetts. While all racial groups experienced decreased access to outpatient care owing to the surge in COVID-19 cases from March to June 2020, Asian patients had reduced access to any care (in-person and telehealth) and a decreased rate of access to telehealth services than those of other racial groups. The x-axis represents weeks since March 2020.
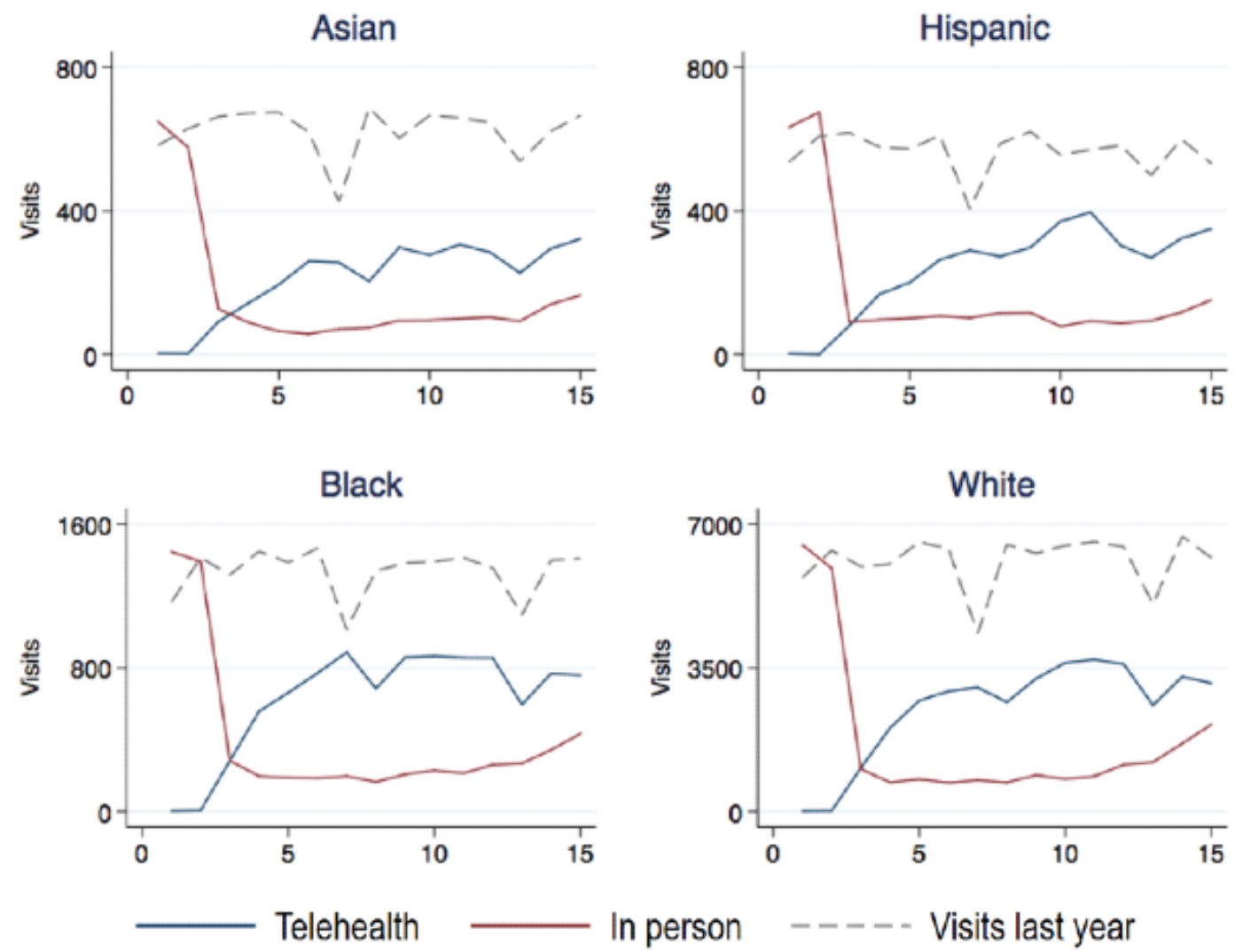

Older patients accessed more outpatient care than younger patients $(P<.001)$ (Table 1$)$. Differences were observed by age and race; differences were most prominent among Asian patients $(P<.001)$. Asian and Hispanic patients aged under 65 years used

telehealth less than age-matched White patients $(P<.001)$, but there was no difference in telehealth usage between Black and White patients under 65 years of age $(P=.07)$. 
In a subgroup analysis conducted among patients who sought care after April 27, we found that older adults were less likely to use video technology ( $19 \%$ of telehealth visits) rather than the telephone compared to their younger counterparts $(26 \%$ of telehealth visits; $P<.001)$. White patients used video technology significantly more ( $25 \%$ of telehealth visits) than those of all other races (Black patients: $16 \%$, Hispanic patients: $23 \%$, and Asian patients: $17 \% ; P<.001)$.

Table 1. Overall usage of in-person and telehealth services from March 2 to June 10, 2020, at a single academic medical center in Boston, Massachusetts, among patients stratified by age and race, recorded at the time of registration $(\mathrm{N}=129,844)$.

\begin{tabular}{|c|c|c|c|c|}
\hline Parameters & Total & In-person visits & Telehealth visits & $P$ value \\
\hline Age (years), n (\%) & & & & $<.001$ \\
\hline $18-29$ & $11,121(100)$ & $5179(46.6)$ & $5942(53.4)$ & \\
\hline $30-39$ & $19,675(100)$ & $10,234(52.0)$ & $9441(48.0)$ & \\
\hline $40-49$ & $15,831(100)$ & $6623(41.8)$ & $9208(58.2)$ & \\
\hline $50-59$ & $23,238(100)$ & 9437 (40.6) & $13,801(59.4)$ & \\
\hline $60-69$ & $28,120(100)$ & $11,535(41.0)$ & $16,585(59.0)$ & \\
\hline $70-79$ & $20,972(100)$ & 7914 (37.7) & $13,058(62.3)$ & \\
\hline$\geq 80$ & $10,887(100)$ & $4076(37.4)$ & $6811(62.6)$ & \\
\hline Race, $\mathrm{n}(\%)$ & & & & $<.001$ \\
\hline Asian & $5661(100)$ & $2499(44.1)$ & $3162(55.9)$ & \\
\hline Black & $15,423(100)$ & $6009(39.0)$ & $9414(61.0)$ & \\
\hline Hispanic & $6243(100)$ & $2656(42.5)$ & 3587 (57.5) & \\
\hline Other & $8499(100)$ & 3817 (44.9) & $4692(55.1)$ & \\
\hline Unknown & $30,621(100)$ & $14,240(46.5)$ & $16,381(53.5)$ & \\
\hline White & $63,397(100)$ & $25,777(40.7)$ & $37,620(59.3)$ & \\
\hline Total, n (\%) & $129,844(100)$ & $54,998(42.4)$ & $74,846(57.6)$ & \\
\hline
\end{tabular}

${ }^{\mathrm{a}} \mathrm{N} / \mathrm{A}$ : not applicable.

\section{Discussion}

\section{Principal Findings}

Our results indicate that implementation of telehealth does not necessarily exacerbate the inequity in health care access, but it should be monitored carefully. Patients had fewer ambulatory visits of any kind during the COVID-19 pandemic; however, Asian and White patients accessed care less than Black and Hispanic patients compared to baseline. Black and White patients accessed telehealth care more than Hispanic and Asian patients. Patients with an "unknown" racial or ethnic designation upon registration had the least access to care.

Contrary to concerns that older patients might have difficulty navigating technology [6], our older patients used telehealth more often than younger patients. The potential reasons for this could be that younger patients perceived their clinical needs to be less urgent, had variable awareness of telehealth services, or experienced additional barriers [7]. However, we found that among telehealth users, patients over 65 years of age were less likely to use video technology, which may reflect concerns with technology; these concerns have been explored by other investigators [6,8]. Similarly, Black, Asian, and Hispanic patients were less likely to use video technology than White patients.

\section{Limitations}

The limitations of this study include the use of administrative data and self-reported racial designations; approximately $22 \%$ of patients in our study belonged to an "unknown" racial category, which may limit our ability to draw inferences $[9,10]$. Further, our documentation of telehealth early in the pandemic was unable to distinguish telephonic and video visits, which may vary across racial and age groups [6].

\section{Conclusions}

In conclusion, after a rapid increase in telehealth use at a single academic medical center in Boston, Massachusetts, we observed variable engagement of our patient population by both race and age in telehealth, but the trends did not mirror previously described patterns of health access disparity.

\section{Acknowledgments}

JPS is supported by award\# K08HS024288 from the Agency for Healthcare Research and Quality. The content of this paper is solely the responsibility of the authors and does not represent the official views of the Agency for Healthcare Research and Quality. 


\section{Conflicts of Interest}

None declared.

\section{References}

1. Shachar C, Engel J, Elwyn G. Implications for Telehealth in a Postpandemic Future: Regulatory and Privacy Issues. JAMA 2020 Jun 16;323(23):2375-2376. [doi: 10.1001/jama.2020.7943] [Medline: $\underline{\text { 32421170] }}$

2. Kruse CS, Krowski N, Rodriguez B, Tran L, Vela J, Brooks M. Telehealth and patient satisfaction: a systematic review and narrative analysis. BMJ Open 2017 Aug 03;7(8):e016242 [FREE Full text] [doi: 10.1136/bmjopen-2017-016242] [Medline: 28775188]

3. Rodriguez JA, Clark CR, Bates DW. Digital Health Equity as a Necessity in the 21st Century Cures Act Era. JAMA 2020 Jun 16;323(23):2381-2382. [doi: 10.1001/jama.2020.7858] [Medline: 32463421]

4. Ramsetty A, Adams C. Impact of the digital divide in the age of COVID-19. J Am Med Inform Assoc 2020 Jul 01;27(7):1147-1148 [FREE Full text] [doi: 10.1093/jamia/ocaa078] [Medline: 32343813]

5. Lin CC, Dievler A, Robbins C, Sripipatana A, Quinn M, Nair S. Telehealth In Health Centers: Key Adoption Factors, Barriers, And Opportunities. Health Aff (Millwood) 2018 Dec;37(12):1967-1974. [doi: 10.1377/hlthaff.2018.05125] [Medline: $\underline{30633683}$ ]

6. Fischer SH, Ray KN, Mehrotra A, Bloom EL, Uscher-Pines L. Prevalence and Characteristics of Telehealth Utilization in the United States. JAMA Netw Open 2020 Oct 01;3(10):e2022302 [FREE Full text] [doi:

10.1001/jamanetworkopen.2020.22302] [Medline: 33104208]

7. Welch BM, Harvey J, O'Connell NS, McElligott JT. Patient preferences for direct-to-consumer telemedicine services: a nationwide survey. BMC Health Serv Res 2017 Nov 28;17(1):784. [doi: 10.1186/s12913-017-2744-8] [Medline: 29183372]

8. Kruse C, Fohn J, Wilson N, Nunez Patlan E, Zipp S, Mileski M. Utilization Barriers and Medical Outcomes Commensurate With the Use of Telehealth Among Older Adults: Systematic Review. JMIR Med Inform 2020 Aug 12;8(8):e20359 [FREE Full text] [doi: 10.2196/20359] [Medline: 32784177]

9. Labgold K, Hamid S, Shah S, Gandhi NR, Chamberlain A, Khan F, et al. Measuring the missing: greater racial and ethnic disparities in COVID-19 burden after accounting for missing race/ethnicity data. medRxiv. Preprint posted online October 2, 2020. [doi: 10.1101/2020.09.30.20203315]

10. Long JA, Bamba MI, Ling B, Shea JA. Missing race/ethnicity data in Veterans Health Administration based disparities research: a systematic review. J Health Care Poor Underserved 2006 Feb;17(1):128-140. [doi: 10.1353/hpu.2006.0029] [Medline: 16520522]

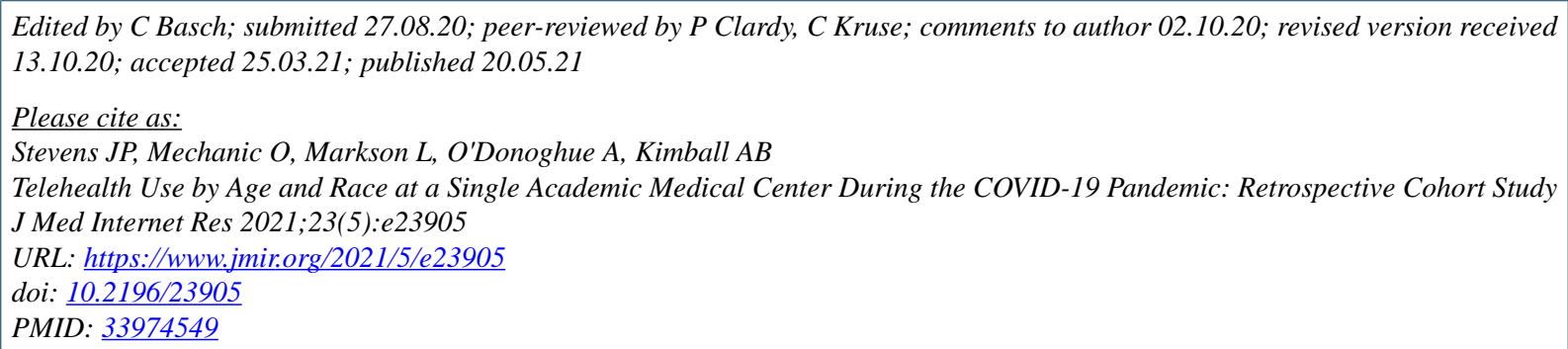

CJennifer P Stevens, Oren Mechanic, Lawrence Markson, Ashley O'Donoghue, Alexa B Kimball. Originally published in the Journal of Medical Internet Research (https://www.jmir.org), 20.05.2021. This is an open-access article distributed under the terms of the Creative Commons Attribution License (https://creativecommons.org/licenses/by/4.0/), which permits unrestricted use, distribution, and reproduction in any medium, provided the original work, first published in the Journal of Medical Internet Research, is properly cited. The complete bibliographic information, a link to the original publication on https://www.jmir.org/, as well as this copyright and license information must be included. 